\title{
Partitions of compact Hausdorff spaces
}

\author{
by
}

Gary Gruenhage (Auburn, Ala.)

\begin{abstract}
Under the assumption that the real line cannot be covered by $\omega_{1}$-many nowhere dense sets, it is shown that (a) no Čech-complete space can be partitioned into $\omega_{1}$-many closed nowhere dense sets; (b) no Hausdorff continuum can be partitioned into $\omega_{1}$-many closed sets; and (c) no compact Hausdorff space can be partitioned into $\omega_{1}$-many closed $G_{\delta}$-sets.
\end{abstract}

Introduction. W. Sierpiński [S] proved in 1918 that no Hausdorff continuum (i.e., compact connected space) can be partitioned into countably many closed sets. On the other hand, it is trivial to find continua (e.g., the unit interval!) which can be partitioned into $\omega_{1}$-many closed sets if the continuum hypothesis $(\mathrm{CH})$ is assumed. This led Steve Watson [W] to ask if there is a ZFC example (i.e., one which does not require any special axioms of set theory):

QUESTION 1. Is there, in ZFC, a Hausdorff continuum which is the union of $\omega_{1}$-many disjoint closed sets?

Another partition problem concerning compact Hausdorff spaces is due to Dow and Porter [DP]. Motivated by Arkhangel'skiil's result $\left[\mathrm{A}_{1}\right]$ that a first-countable compact Hausdorff space is either countable or has cardinality $c$, they investigate the possible cardinalities of first-countable $H$-closed spaces. (A space is $H$-closed if it is closed in every Hausdorff space in which it is embedded.) They show that the existence of a first-countable $H$-closed space of cardinality $\lambda$ is equivalent to the existence of a compact Hausdorff space which can be partitioned into $\lambda$-many closed $G_{\delta}$-sets, and point out that it is consistent with $c>\omega_{1}$ that there is a first-countable $H$-closed space of cardinality $\omega_{1}$. They leave open the possibility of a ZFC example of such a space:

Partially supported by National Science Foundation grant NSF-DMS-9102725. 
QUESTION 2. Is there, in ZFC, a compact Hausdorff space which can be partitioned into $\omega_{1}$-many closed $G_{\delta}$-sets? (Equivalently, is there in ZFC a first-countable $H$-closed space of cardinality $\omega_{1}$ ? $)\left({ }^{1}\right)$

Finally, we consider partitions of compact Hausdorff spaces (and, more generally, Čech-complete spaces) into closed nowhere dense sets. The Baire category theorem says there are no countable such partitions, even ignoring the adjective "closed". What about partitions of size $\omega_{1}$ ?

QUESTION 3. Is there, in ZFC, a compact Hausdorff (or Čech-complete) space which can be partitioned into $\omega_{1}$-many closed nowhere dense sets?

The Štěpánek-Vopěnka Theorem [SV] says that any nowhere separable metric space is the union of not more than $\omega_{1}$-many nowhere dense sets. This theorem implies, e.g., that the complete metric space $\omega_{1}^{\omega}$, where $\omega_{1}$ carries the discrete topology, can be partitioned into $\omega_{1}$-many nowhere dense sets; but the members of the partition given by the proof are not closed. There are also ZFC examples of compact Hausdorff spaces which can be partitioned into $\omega_{1}$-many nowhere dense sets (folklore, I believe; see also Example 3.4), but again members of the partition are not closed. So requiring the members of the partition to be closed is crucial for Question 3 . Note that the adjective "nowhere dense" is also crucial, because there are of course compact Hausdorff spaces and complete metric spaces of cardinality $\omega_{1}$ (the ZFC examples having isolated points).

In this note we show that the answer to Questions 1-3 is "no": there are no such ZFC examples. This follows from:

THEOREM 0.1. If the real line is not the union of $\omega_{1}$-many nowhere dense sets, then:

(a) no Čech-complete (e.g., compact Hausdorff or completely metrizable) space is the union of $\omega_{1}$-many disjoint closed nowhere dense sets;

(b) no Hausdorff continuum is the union of $\omega_{1}$-many disjoint closed sets;

(c) no compact Hausdorff space is the union of $\omega_{1}$-many disjoint closed $G_{\delta}$-sets.

Thus (a), (b), and (c) hold in, e.g., any model of Martin's Axiom plus the negation of the continuum hypothesis. The proof of (a) and (b) does not extend to higher cardinals, but we do not know if the results do. In particular, we do not know if there is a ZFC example of a Hausdorff continuum which can be partitioned into $\omega_{2}$-many closed sets, or a compact Hausdorff space which can be partitioned into $\omega_{2}$-many closed nowhere dense sets. See Section 3 for more discussion of these and other questions.

$\left.{ }^{1}\right)$ I am indebted to Doug Mooney and Jack Porter for informing me of [DP] and this problem. 
However, (c) does extend to higher cardinals, as does (a) in some special cases.

THEOREM 0.2. If the real line is not the union of $\kappa$-many nowhere dense sets, then

(d) no compact Hausdorff space is the union of $\lambda$-many disjoint closed $G_{\delta}$-sets, where $\omega_{1} \leq \lambda \leq \kappa$;

(e) no compact ordered space is the union of $\leq \kappa$-many disjoint closed nowhere dense sets;

(f) no Čech-complete space with a dense set of $G_{\delta}$-points (e.g., no completely metrizable space) is the union of $\leq \kappa$-many disjoint closed nowhere dense sets.

1. Partitions into closed nowhere dense sets and closed $G_{\delta}$-sets. In this section we prove Theorem 0.2 , and all of Theorem 0.1 except for part (b), which is postponed until the next section.

Recall that a space $X$ is $\check{C}$ ech-complete if it is a $G_{\delta}$-set in its Stone-Cech compactification $\beta X$; equivalently, there is a sequence $\mathcal{U}_{n}, n<\omega$, of open covers of $X$ such that if $K_{n}, n<\omega$, is a decreasing sequence of closed sets with $K_{n} \subseteq U_{n} \in \mathcal{U}_{n}$ for each $n$, then $K_{\omega}=\bigcap_{n<\omega} K_{n}$ is compact and the $K_{n}$ 's form an outer network for $K_{\omega}$ (i.e., every open set containing $K_{\omega}$ contains some $K_{n}$ ). Compact Hausdorff spaces are Cech-complete, and a metrizable space is completely metrizable iff it is Čech-complete. Čechcomplete spaces are Baire spaces, and are hereditary with respect to closed subsets and $G_{\delta}$-subsets.

For the sake of completeness, we include a proof of the following essentially known and elementary lemma:

LEMMA 1.1. Let $\mathcal{U}_{n}, n<\omega$, be a sequence of open covers of $X$ which witness that $X$ is $\check{C}$ ech-complete. Then each non-empty open subset of $X$ contains a compact $G_{\delta}$-set $L$ which, for each $n$, is contained in some member of $\mathcal{U}_{n}$.

Proof. Let $V$ be a non-empty open subset of $X$. Inductively define a sequence $V_{n}, n<\omega$, of open sets with $\operatorname{cl}\left(V_{n+1}\right) \subseteq V_{n} \subseteq U_{n} \in \mathcal{U}_{n}$ for each $n$. Then $L=\bigcap_{n<\omega} V_{n}$ is the desired set.

THEOREM 1.2. If the real line is not the union of $\omega_{1}$-many nowhere dense sets, then no Cech-complete space is the union of $\omega_{1}$-many disjoint closed nowhere dense sets.

P r o of. Suppose the Čech-complete space $X$ is the union of (non-empty) disjoint closed nowhere dense sets $C_{\alpha}, \alpha<\omega_{1}$. Call a subset $A$ of $X$ big if $A$ meets uncountably many $C_{\alpha}$ 's; otherwise $A$ is small. 
We first establish that every (non-empty) open subset of $X$ contains a (non-empty) small closed $G_{\delta}$-set. (Henceforth let us assume that named subsets of $X$ are non-empty unless stated otherwise.) Suppose $V$ is an open set which does not contain such. Let $K_{0}$ be a compact $G_{\delta}$-subset of $V$. Then $K_{0}$ is big; hence $K_{0} \backslash C_{0}$ contains a compact $G_{\delta}$-set $K_{1}$. Similarly, $K_{1}$ contains a compact $G_{\delta}$-set $K_{2}$ missing $C_{1}, K_{2}$ contains a compact $G_{\delta^{-}}$set $K_{3}$ missing $C_{2}$, and so forth. Let $K_{\omega}=\bigcap_{n<\omega} K_{n}$. Then $K_{\omega}$ is also a compact $G_{\delta}$-set, hence contains a compact $G_{\delta}$-set $K_{\omega+1}$ missing $C_{\omega}$. Clearly we can continue on to construct a decreasing sequence $K_{\alpha}, \alpha<\omega_{1}$, of compact $G_{\delta^{-}}$ sets such that $K_{\alpha+1}$ misses $C_{\alpha}$ for each $\alpha<\omega_{1}$. Then $\bigcap\left\{K_{\alpha}: \alpha<\omega_{1}\right\}=\emptyset$, contradiction.

Let $\left\{\mathcal{U}_{n}\right\}_{n<\omega}$ be a collection of open covers of $X$ that witness that $X$ is Cech-complete; for convenience, assume $\mathcal{U}_{0}=\{X\}$. Let $\mathcal{K}_{n}$ be the collection of all closed subsets of $X$, with non-empty interiors, that are contained in some member of $\mathcal{U}_{n}$.

Let $K \in \mathcal{K}_{n}$. Let $L_{0}$ and $R_{0}$ be disjoint members of $\mathcal{K}_{n+1}$ contained in $K$. Then $\operatorname{int}\left(L_{0}\right)$ contains a small compact $G_{\delta}$-set $M$. Since the traces of the $\mathcal{U}_{n}$ 's on $M$ witness that $M$ is Cech-complete, by Lemma 1.1, $M$ contains a (small) compact $G_{\delta}$-set $N$ which, for each $j$, is contained in some member of $\mathcal{U}_{j}$. For $j>0$, one can find disjoint $L_{j}, L_{j}^{\prime} \in \mathcal{K}_{n+j+1}$ with $L_{j} \cup L_{j}^{\prime} \subseteq L_{j-1}$, and $\bigcap_{j<\omega} L_{j}=N$. As $N$ meets only countably many $C_{\alpha}$ 's, one can now define disjoint $R_{j}, R_{j}^{\prime} \in \mathcal{K}_{n+j+1}$ with $R_{j} \cup R_{j}^{\prime} \subseteq R_{j-1}$ such that $C_{\alpha} \cap\left(\bigcap_{j<\omega} R_{j}\right)=\emptyset$ whenever $C_{\alpha} \cap N \neq \emptyset$.

Now we use the construction of the previous paragraph repeatedly to define $\left\{K_{\sigma}: \sigma \in 2^{<\omega}\right\}$ satisfying:

(a) $n=\operatorname{dom} \sigma \Rightarrow K_{\sigma} \in \mathcal{K}_{n}$;

(b) $\sigma \subseteq \tau \Rightarrow K_{\sigma} \supseteq K_{\tau}$;

(c) if $\sigma \in 2^{<\omega}$, there is some $\tau \supseteq \sigma$ such that, if

$$
C_{\alpha} \cap K_{\tau^{\wedge}\langle 0\rangle} \cap K_{\tau^{\wedge}\langle 0,0\rangle} \cap \ldots \neq \emptyset,
$$

then

$$
C_{\alpha} \cap K_{\tau^{\wedge}\langle 1\rangle} \cap K_{\tau^{\wedge}\langle 1,1\rangle} \cap \ldots=\emptyset .
$$

To start, let $K_{\emptyset}=X$. Let $L_{n}, L_{n}^{\prime}, R_{n}, R_{n}^{\prime}$ be as above with $K=K_{\emptyset}$. If $n>0$ and $\tau \in \omega^{n}$ is constant 0 (resp., 1), let $K_{\tau}=L_{n-1}$ (resp., $R_{n-1}$ ), and let $K_{\tau^{\wedge}\langle 1\rangle}$ (resp., $\left.K_{\tau^{\wedge}\langle 0\rangle}\right)$ be $L_{n}^{\prime}$ (resp., $R_{n}^{\prime}$ ).

At this point, for each $\sigma \in 2^{<\omega}$, either $K_{\sigma}$ is undefined, or $K_{\sigma}, K_{\sigma^{\wedge}\langle 0\rangle}$, and $K_{\sigma^{\wedge}\langle 1\rangle}$ are all defined, or $K_{\sigma}$ is defined but neither $K_{\sigma^{\wedge}}\langle 0\rangle$ nor $K_{\sigma^{\wedge}}\langle 1\rangle$ are defined. Call $\sigma$ 's with the latter property ends. Call $\emptyset$ the initial base.

Let $m$ be least such that some $\varrho \in \omega^{m}$ is an end. Call $\varrho$ the new base, and define $K_{\sigma}$ for extensions $\sigma$ of $\varrho$ as we did above for $\emptyset$. More precisely, let $L_{n}, L_{n}^{\prime}, R_{n}, R_{n}^{\prime}$ be as above with $K=K_{\varrho}$. If $n>0$ and $\tau \in \omega^{n}$ is constant 0 
(resp., 1), let $K_{\varrho^{\wedge} \tau}=L_{n-1}$ (resp., $\left.R_{n-1}\right)$, and let $K_{\varrho^{\wedge} \tau^{\wedge}\langle 1\rangle}\left(\operatorname{resp} ., K_{\varrho^{\wedge} \tau^{\wedge}\langle 0\rangle}\right)$ be $L_{n}^{\prime}$ (resp., $R_{n}^{\prime}$ ).

Note that all ends other than $\varrho$ are still ends, and of course there are many more ends. Continue in like manner, each time choosing as the new base an end with least possible domain, until $K_{\sigma}$ is defined for all $\sigma$. Note that each end eventually becomes the new base, and that at each stage, if $K_{\sigma}$ is first defined at that stage, then either $\sigma, \sigma^{\wedge}\langle 0\rangle$, or $\sigma^{\wedge}\langle 1\rangle$ is an end. It follows that conditions (a)-(c) above are satisfied.

For each $f \in 2^{\omega}$, let $K_{f}=\bigcap_{n<\omega} K_{f \mid n}$. For $\alpha<\omega_{1}$, let

$$
N_{\alpha}=\left\{f \in 2^{\omega}: K_{f} \cap C_{\alpha} \neq \emptyset\right\} .
$$

Since the $K_{f \mid n}$ 's form an outer network for $K_{f}$, it follows that each $N_{\alpha}$ is closed in $2^{\omega}$. Since each $K_{f}$ is non-empty, and the $C_{\alpha}$ 's cover $X, 2^{\omega}=$ $\bigcup\left\{N_{\alpha}: \alpha<\omega_{1}\right\}$.

We arrive at a contradiction by showing that each $N_{\alpha}$ is nowhere dense. (This is a contradiction because, if the Cantor set is the union of $\kappa$-many nowhere dense sets, so is $\mathbb{R}$ - to see this, note that both have a co-countable subset homeomorphic to the irrationals.) Suppose on the contrary that $\operatorname{int}\left(N_{\alpha}\right) \neq \emptyset$ for some $\alpha$. Then for some $\sigma \in 2^{\omega}, K_{f} \cap C_{\alpha} \neq \emptyset$ whenever $\sigma \subseteq f$. Let $\tau$ be an extension of $\sigma$ guaranteed by (c) above. By the conclusion of (c), $K_{f} \cap C_{\alpha}=\emptyset$ for some $f \supseteq \tau$. This contradiction completes the proof.

A number of similar results may be proved using minor modifications of the above technique. We will use the following lemma, which is essentially a statement of the hypotheses necessary to carry out the Cantor tree construction of the above proof.

Lemma 1.3. Let $\mathcal{C}$ be a closed cover of the space $X$. Suppose there are collections $\mathcal{K}_{n}, n<\omega$, of non-empty closed subsets of $X$ satisfying:

(I) $X \in \mathcal{K}_{0}$;

(II) if $K_{n} \in \mathcal{K}_{n}$ for each $n$, and $\left\{K_{n}\right\}_{n<\omega}$ is decreasing, then $\left\{K_{n}\right\}_{n<\omega}$ is an outer network for the set $K_{\omega}=\bigcap_{n<\omega} K_{n}$;

(III) if $K \in \mathcal{K}_{n}$, then there exist disjoint $L_{0}, R_{0} \subseteq K$, and disjoint $L_{j}, L_{j}^{\prime}, R_{j}, R_{j}^{\prime} \in K_{n+j+1}$ for $j>0$, such that:

(i) $L_{j}, L_{j}^{\prime}, R_{j}, R_{j}^{\prime} \in \mathcal{K}_{n+j+1}$ for $j \in \omega$;

(ii) $L_{j} \cup L_{j}^{\prime} \subseteq L_{j-1}$ and $R_{j} \cup R_{j}^{\prime} \subseteq R_{j-1}$ for $j>0$;

(iii) if $C \in \mathcal{C}$ and $C \cap \partial L_{\omega} \neq \emptyset$, then $C \cap \partial K_{\omega}=\emptyset$.

Then the real line $\mathbb{R}$ is the union of $\leq|\mathcal{C}|$ nowhere dense sets.

Proof. As in the proof of Theorem 1.2 , use the $\mathcal{K}_{n}$ 's to construct $K_{\sigma}$ 's, $\sigma \in 2^{<\omega}$, satisfying:

(a) $\operatorname{dom} \sigma=n \Rightarrow K_{\sigma} \in \mathcal{K}_{n}$; 
(b) $\sigma \subseteq \tau \Rightarrow K_{\sigma} \supseteq K_{\tau}$;

(c) if $\sigma \in 2^{<\omega}$, there is some $\tau \supseteq \sigma$ such that, if

$$
C \cap \partial\left(K_{\tau^{\wedge}\langle 0\rangle} \cap K_{\tau^{\wedge}\langle 0,0\rangle} \cap \ldots\right) \neq \emptyset,
$$

then

$$
C \cap \partial\left(K_{\tau^{\wedge}\langle 1\rangle} \cap K_{\tau^{\wedge}\langle 1,1\rangle} \cap \ldots\right)=\emptyset .
$$

For each $C \in \mathcal{C}$, let

$$
N(C)=\left\{f \in 2^{\omega}: C \cap \partial K_{f} \neq \emptyset\right\} .
$$

If $f \notin N(C)$, then $C \cap\left(K_{f \mid n} \backslash K_{\omega}\right)=\emptyset$ for some $n \in \omega$. Then $C \cap \partial K_{g}=\emptyset$ for all $g \supseteq f \mid n$; hence each $N(C)$ is closed. The proof that each $N(C)$ is nowhere dense and that $2^{\omega}=\bigcup\{N(C): C \in \mathcal{C}\}$ is the same as before.

Our first use of the lemma will be to show

THEOREM 1.4. If the real line is not the union of $\kappa$ nowhere dense sets, then no Čech-complete space with a dense set of $G_{\delta}$-points (in particular, no completely metrizable space) is the union of $\kappa$ disjoint closed nowhere dense sets.

P r o o f. Suppose $\mathcal{C}$ is a disjoint cover of cardinality $\kappa$ of a Čech-complete space $X$ by closed nowhere dense sets. Let $\mathcal{K}_{n}$ be as in the proof of Theorem 1.2. Since members of $\mathcal{K}_{n}$ have non-empty interiors, they contain $G_{\delta}$-points and hence small closed $G_{\delta}$-sets. Thus $L_{j}$ 's and $R_{j}$ 's satisfying the conditions of Lemma 1.3 may be constructed in the same way as in Theorem 1.2. By Lemma 1.3, the real line is the union of $\leq|\mathcal{C}|$ nowhere dense sets, contradiction.

THEOREM 1.5. If the real line is not the union of $\kappa$-many nowhere dense sets, and $\omega<\lambda \leq \kappa$, then no compact Hausdorff space is the union of $\lambda$-many closed $G_{\delta}$-sets.

Pr o of. Suppose $\mathcal{C}$ is a partition of the compact Hausdorff space $X$ into $\lambda$ closed $G_{\delta}$-sets, $\omega<\lambda \leq \kappa$. We may assume that $\lambda$ is the least cardinal of any uncountable partition of any compact Hausdorff space into closed $G_{\delta}$-sets. Let $\mathcal{K}$ be the collection of all big closed subsets of $X$, where a set is called big if it meets $\lambda$-many members of $\mathcal{C}$. Let $\mathcal{K}_{n}=\mathcal{K}$ for all $n$. The theorem will follow if we can show that $\mathcal{C}$ and $\left\{\mathcal{K}_{n}\right\}_{n<\omega}$ satisfy the conditions of Lemma 1.3. Conditions (I) and (II) are obvious.

We show (III) holds. Let $K \in \mathcal{K}$. Since $K$ is compact and big, there is $C^{\#} \in \mathcal{C}$ such that every neighborhood of $C^{\#} \cap K$ in the subspace $K$ is big. Clearly, we can construct a descending sequence $\left\{U_{n}\right\}_{n<\omega}$ of relatively open (in $K$ ) neighborhoods of $C^{\#} \cap K$ satisfying:

(i) $\bigcap_{n<\omega} U_{n}=C^{\#} \cap K$;

(ii) $\operatorname{cl}\left(U_{n+1}\right) \subseteq U_{n}$ for all $n$; 
(iii) $U_{n} \backslash U_{n+1}$ is big for all $n$.

(Minimality of $\lambda$ is used to obtain (iii).)

Let $L_{0}=\operatorname{cl}\left(U_{2}\right)$ and $K_{0}=\operatorname{cl}\left(U_{0}\right) \backslash U_{1}$. For $j>0$, let $L_{j}=\operatorname{cl}\left(U_{3 j+2}\right)$ and $L_{j}^{\prime}=\operatorname{cl}\left(U_{3 j}\right) \backslash U_{3 j+1}$, and let $K_{j}$ and $K_{j}^{\prime}$ be arbitrary disjoint big closed subsets of $K_{j-1}$. Since $L_{\omega}=C^{\#} \cap K$, it is easy to see that these $K$ 's and $L$ 's satisfy condition (III).

A. V. Arkhangel'skiu $\left[\mathrm{A}_{2}\right]$ has shown that no compact Hausdorff space can be partitioned into more than $c$ closed $G_{\delta}$-sets. Thus we have the following corollary:

COROLLARY 1.6. If the real line is not the union of fewer than $c$ nowhere dense sets, then every partition of a compact Hausdorff space into closed $G_{\delta}$-sets is either countable or has cardinality c. (Equivalently, every first countable $H$-closed space is either countable or has cardinality c.)

THEOREM 1.7. If the real line is not the union of $\kappa$ nowhere dense sets, then no compact ordered space is the union of $\leq \kappa$ disjoint closed nowhere dense sets.

P r o of. Apply Lemma 1.3 with $\mathcal{C}$ being an assumed disjoint cover of a compact ordered space $X$ by $\leq \kappa$ closed nowhere dense sets, and each $\mathcal{K}_{n}$ the collection of all non-degenerate closed intervals of $X$. Since the boundaries of intervals meet at most two members of $\mathcal{C}$, the $L_{j}$ 's and $R_{j}$ 's satisfying condition (III) are easily constructed.

2. Partitions of continua. The purpose of this section is to prove

THEOREM 2.1. If the real line is not the union of $\omega_{1}$-many nowhere dense sets, then no Hausdorff continuum is the union of $\omega_{1}$-many disjoint closed sets.

We give two proofs. The first is a reasonably direct application of Lemma 1.3. For the second, we prove the following intermediate result which may be of independent interest; this result is due in part to D. P. Bellamy:

THEOREM 2.2. If there is a Hausdorff continuum which is the union of $\kappa$-many disjoint closed sets, then there is one which is the union of $\kappa$-many disjoint closed nowhere dense sets.

Theorem 2.1 is an immediate corollary of Theorems 2.2 and 1.2.

Proof of Theorem 2.1. Let $\mathcal{C}$ be a disjoint cover of a Hausdorff continuum $X$ by $\omega_{1}$-many closed sets. Call a subcontinuum $K$ of $X$ big if $K$ meets uncountably many members of $\mathcal{C}$. Note that if $K$ is small (= not big), then $K$ is contained in some member of $\mathcal{C}$ (by Sierpiński's theorem). 
FACT 1. For each big subcontinuum $K$ of $X$ and for each finite subcollection $\mathcal{F}$ of $\mathcal{C}$, there are disjoint big subcontinua $L$ and $R$ of $K$ that miss every member of $\mathcal{F}$.

To see this, choose $C(0), C(1) \in \mathcal{C} \backslash \mathcal{F}$ meeting $K$. Let $U(0)$ and $U(1)$ be relatively open sets in $K$ containing $C(0) \cap K$ and $C(1) \cap K$, respectively, whose closures are disjoint and miss $\bigcup \mathcal{F}$. Let $x(e) \in C(e) \cap K, e<2$, and let $M(e)$ be the component of $X(e)$ in $\operatorname{cl}(U(e))$. By [E; Lemma 6.1.25], $M(e)$ meets the boundary of $\operatorname{cl}(U(e))$, hence $M(e)$ is big. Finally, take $L=M(0)$ and $R=M(1)$.

FACT 2. Each big subcontinuum $K$ of $X$ contains a decreasing sequence $\left\{K_{n}\right\}_{n<\omega}$ of big subcontinua such that each $K_{n} \backslash K_{n+1}$ contains a big subcontinuum and $\bigcap_{n<\omega} K_{n}$ is small.

Suppose the big subcontinuum $K$ fails to satisfy the conclusion of Fact 2 . Let $\mathcal{C}=\left\{C_{\alpha}: \alpha<\omega_{1}\right\}$. Use Fact 1 to construct a decreasing sequence $\left\{L_{n}\right\}_{n<\omega}$ of big subcontinua of $K$ such that each $L_{n} \backslash L_{n+1}$ contains a subcontinuum and $L_{n} \cap C_{n}=\emptyset$. Then $L^{\prime}=\bigcap_{n<\omega} L_{n}$ is big, and also does not satisfy the conclusion of Fact 2. Construct a decreasing sequence $\left\{L_{\omega+n}\right\}_{n<\omega}$ of big subcontinua of $L^{\prime}$ such that each $L_{\omega+n} \backslash L_{\omega+n+1}$ contains a big subcontinuum and $L_{\omega+n} \cap C_{\omega+n}=\emptyset$. Clearly we can continue this process, defining a decreasing sequence $\left\{L_{\alpha}: \alpha<\omega_{1}\right\}$ of big subcontinua of $K$ such that $L_{\alpha} \cap C_{\alpha}=\emptyset$. Then $\bigcap\left\{L_{\alpha}: \alpha<\omega_{1}\right\}$ misses every $C \in \mathcal{C}$. This contradiction completes the proof of Fact 2 .

Now let $\mathcal{K}$ be the collection of all big subcontinua of $X$, and let $\mathcal{K}_{n}=\mathcal{K}$ for all $n$. It is easy to see from Facts 1 and 2 that $\mathcal{C}$ and $\mathcal{K}_{n}, n<\omega$, satisfy the conditions of Lemma 1.3; thus the real line is the union of $|\mathcal{C}|=\omega_{1}$ nowhere dense sets.

We now work towards proving Theorem 2.2. A naive attempt towards proving it might go as follows. Suppose $\mathcal{C}$ is a closed partition of a Hausdorff continuum $X$. Remove from $X$ the interiors of all members of $\mathcal{C}$. We might hope that what remains is still connected, and that the traces of the members of $\mathcal{C}$ on the remainder are nowhere dense. Neither of these hopes are justified, but both problems can be fixed. The second problem is fixed by iterating the process until what is left of each $C$ is nowhere dense. We will need the following definition:

Definition 2.3. Let $\mathcal{C}$ be a closed partition of a compact Hausdorff space $X$. If $x \in X$, let $C(x)$ be the member of $\mathcal{C}$ containing $x$. Inductively define $X(\alpha)$ and $\operatorname{rank}(x)$ as follows:

(i) $X(0)=X$;

(ii) $\operatorname{rank}(x)=0$ iff $x \in \operatorname{int}(C(x))$; 
(iii) if $X(\beta)$ and $\operatorname{rank}(x)=\beta$ has been defined for all $\beta<\alpha$, then $X(\alpha)=X \backslash\{x \in X: \operatorname{rank}(x)<\alpha\}$;

(iv) if $x \in X(\alpha)$, then $\operatorname{rank}(x)=\alpha$ iff $x \in \operatorname{int}_{X(\alpha)}[C(x) \cap X(\alpha)]$;

(v) $X(\infty)=\bigcap\{X(\alpha): \alpha$ an ordinal $\}$;

(vi) $\operatorname{rank}(x)=\infty$ iff $x \in X(\infty)$.

For convenience, we say $\alpha<\infty$ for each ordinal $\alpha$.

LEMma 2.4. (a) If $\beta$ is an ordinal, then $\operatorname{rank}(x) \leq \beta$ iff for some neighborhood $N$ of $x, \operatorname{rank}(y)<\beta$ for each $y \in N \backslash C(x)$.

(b) Each $X(\alpha)$ is closed.

(c) For limit $\alpha, X(\alpha)=\bigcap_{\beta<\alpha} X(\beta)$.

(d) There is an ordinal $\alpha$ such that $X(\alpha)=X(\beta)=X(\infty)$ for all $\beta>\alpha$.

(e) For each $C \in \mathcal{C}, C \cap X(\infty)$ is nowhere dense in $X(\infty)$.

Pr o of. Straightforward.

Lemma 2.5. Let $X$ be a compact Hausdorff space, $\mathcal{C}$ a closed partition of $X$, and $Y$ a closed subspace of $X$. Let $y \in Y$. Then the rank of $y$ in $Y$ with respect to the partition $\mathcal{C} \mid Y=\{C \cap Y: C \in \mathcal{C}\}$ is not greater than the rank of $y$ in $X$ with respect to $\mathcal{C}$.

P r o of. Straightforward induction, using Lemma 2.4(a).

The following is a key lemma which shows that, for a continuum, the members of $\mathcal{C}$ will not disappear in the process of removing interiors:

LEMMA 2.6. Let $\mathcal{C}$ be a closed partition of a Hausdorff continuum $X$. Then each $C \in \mathcal{C}$ contains some point $x$ with $\operatorname{rank}(x)=\infty$ (i.e., $C \cap X(\infty)$ $\neq \emptyset)$.

Proof. If not, then we can find some ordinal $\gamma$ and some $C \in \mathcal{C}$ such that $\operatorname{rank}(x)<\gamma$ for each $x \in C$. Assume $\gamma$ is the least such ordinal for any counterexample, i.e., for any Hausdorff continuum and closed partition, each member of the partition has points of rank $\geq \beta$ for any $\beta<\gamma$. Then $\gamma$ is not a limit ordinal (else $X_{\gamma} \cap C=\bigcap_{\beta<\gamma} X_{\beta} \cap C \neq \emptyset$, and $C$ contains a point of rank $\geq \gamma)$. Let $\gamma=\alpha+1$. By Lemma 2.4(a), there is a closed neighborhood $N$ of $C$ such that $\operatorname{rank}(x)<\alpha$ for any point $x \in N \backslash C$. Choose $p \in C$, and let $Y$ be the component of $p$ in $N$. By [E; Lemma 6.1.25], $Y \cap \partial N \neq \emptyset$. Thus $Y \cap C^{\prime} \neq \emptyset$ for some $C^{\prime} \in \mathcal{C}, C^{\prime} \neq C$. By Lemma 2.5, each point of $Y \cap C^{\prime}$ has rank $<\alpha$ in $Y$ with respect to $\mathcal{C} \mid Y$. This contradicts the minimality of $\gamma$.

Proof of Theorem 2.2. Let $X$ be a Hausdorff continuum, and $\mathcal{C}$ a closed partition of $X$. Let

$$
Z=\left\{(x, y) \in X^{2}: \operatorname{rank}(x)=\infty \text { or } \operatorname{rank}(y)=\infty\right\} .
$$


By Lemma 2.6, $\mathcal{D}=\left\{\left(C_{1} \times C_{2}\right) \cap Z: C_{1}, C_{2} \in \mathcal{C}\right\}$ is a partition of $Z$ into non-empty closed sets. That $Z$ is connected follows from the fact that $Z=\bigcup\{(\{x\} \times X) \cup(X \times\{x\}): \operatorname{rank}(x)=\infty\}$.

It remains to show that each member of $\mathcal{D}$ is nowhere dense in $Z$. Suppose on the contrary that $\left(x_{1}, x_{2}\right) \in\left(U_{1} \times U_{2}\right) \cap Z \subseteq\left(C_{1} \times C_{2}\right) \cap Z$, where $U_{1}$ and $U_{2}$ are open in $X$. By Lemma 2.4(e), there is a point $y \in U_{1} \backslash C_{1}$ with $\operatorname{rank}(y)=\infty$. Then $\left(y, x_{2}\right) \in\left[\left(U_{1} \times U_{2}\right) \cap Z\right] \backslash\left[\left(C_{1} \times C_{2}\right) \cap Z\right]$, contradiction.

3. Questions, remarks, and an example. The following question was mentioned in the introduction:

QUESTION 3.1. Is there in ZFC a compact Hausdorff space (or Hausdorff continuum) that can be partitioned into $\omega_{2}$ closed nowhere dense sets? (We do not know the answer for any $\omega_{\alpha}, \alpha \geq 2$, either.)

It is conceivable that the answer could be "yes" for the compact Hausdorff case but "no" for the continuum case. We note also that any model in which there is no such $\omega_{2}$-partitionable compact Hausdorff space (resp., Hausdorff continuum) must also be a model with no $\omega_{1}$-partitionable ones, because of

THEOREM 3.2. Let $X$ be a compact Hausdorff space which can be partitioned into $\kappa$ nowhere dense closed sets, and let $p \in X$. Let $\lambda>\kappa$. Then $Y=X \times(\lambda+1)$ and $Z=Y /[\{p\} \times(\lambda+1)]$ can be partitioned into $\lambda$-many nowhere dense closed sets. If $X$ is connected, so is $Z$.

Pr o of. Straightforward.

QUESTION 3.3. Is it true that some compact Hausdorff space (resp., Hausdorff continuum) can be partitioned into $\omega_{1}$-many closed nowhere dense sets (resp., closed sets) if and only if the unit interval $[0,1]$ can be so partitioned?

By Theorem 1.2, any model in which there is a compact Hausdorff space which is partitionable into $\omega_{1}$-many closed nowhere dense sets, yet $[0,1]$ is not so partitionable, would have to satisfy the following statement:

$(*) \quad[0,1]$ is the union of $\omega_{1}$-many nowhere dense sets, but is not the union of $\omega_{1}$-many disjoint closed sets.

Miller $[\mathrm{M}]$ has constructed a model of $(*)$. We do not know if there is a compact Hausdorff space in Miller's model which is partitionable into $\omega_{1}$-many closed nowhere dense sets.

Fremlin and Shelah [FS] showed that $[0,1]$ is the union of $\omega_{1}$-many nowhere dense sets iff $[0,1]$ can be partitioned into $\omega_{1}$-many (nowhere dense) $G_{\delta}$-sets. This suggests the question (asked by Fremlin in conversation with the author) whether $[0,1]$ not the union of $\omega_{1}$-many nowhere dense sets 
implies that no compact Hausdorff space can be partitioned into $\omega_{1}$-many nowhere dense $G_{\delta}$-sets. The following example shows that the answer is "no".

EXAMPLE 3.4. There is a Hausdorff continuum $X$ which is the union of $\omega_{1}$-many disjoint nowhere dense $G_{\delta}$-sets. (Added in proof. S. Todorčević has kindly pointed out to the author that an example with these properties is given by Theorem 6.16 of [T].)

P r o of. Let $T$ be an Aronszajn tree such that each node has (countably) infinitely many successors (recall that a tree $T$ is Aronszajn if its height is $\omega_{1}$, each level is countable, and it has no uncountable chains; see, e.g., $[\mathrm{K}$; Theorem 5.9]). Call $p \subseteq T$ a path if $p$ is a totally ordered subset of $T$ such that $\{s \in T: s<t\} \subseteq p$ whenever $t \in p$. Let $Y=\left\{\chi_{p}: p\right.$ is a path $\}$ viewed as a subspace of $2^{T}$, where $\chi_{p}$ is the characteristic function of $p$. Then $Y$ is closed in $2^{T}$, hence is compact. Note that a typical basic (cl)open set containing $\chi_{p}$ can be obtained as follows: Let $t \in p$, and let $F$ be a finite subset of $T \backslash p$; then $V(t, F)=\left\{\chi_{q}: t \in q\right.$ and $\left.q \cap F=\emptyset\right\}$ is a clopen neighborhood of $p$. Note also that because each node of $T$ has infinitely many successors, it follows that $Y$ has no isolated points. (Considering this "space of paths" of a tree is due to Todorčević [T], and is closely related to a topology on trees considered by Nyikos [N].)

For $\alpha<\omega_{1}$, let $L_{\alpha}=\left\{\chi_{p}: p\right.$ has order-type $\left.\alpha\right\}$. Clearly, $L_{\alpha}$ is nowhere dense. Note that each $\bigcup_{\beta<\alpha} L_{\beta}$ is closed in $Y$, hence each $\bigcup_{\beta>\alpha} L_{\beta}$ is a $G_{\delta}$-set. Also, $\bigcup_{\beta>\alpha} L_{\beta}$ is the union of the countable collection $\{V(t, \emptyset)$ : $\{s \in T: s<t\}$ has order-type $\alpha\}$ of clopen sets. It follows that each $L_{\alpha}$ is $G_{\delta}$.

Finally, let $X=Y \times[0,1] / Y \times\{1\}$ (i.e., $X$ is the cone over $Y$ ). Then $X$ is a Hausdorff continuum, and $\{Y \times\{1\}\} \cup\left\{L_{\alpha} \times[0,1): \alpha<\omega_{1}\right\}$ is a partition of $X$ into $\omega_{1}$-many nowhere dense $G_{\delta}$-sets.

\section{References}

$\left[\mathrm{A}_{1}\right] \quad$ A. V. Arkhangel'skiǔ , On the cardinality of bicompacta satisfying the first axiom of countability, Soviet Math. Dokl. 10 (1969), 951-955.

$\left[\mathrm{A}_{2}\right]-$, Theorems on the cardinality of families of sets in compact Hausdorff spaces, ibid. 17 (1976), 213-217.

[DP] A. Dow and J. Porter, Cardinalities of H-closed spaces, Topology Proc. 7 (1982), $27-50$.

[E] R. Engelking, General Topology, Heldermann, Berlin 1989.

[FS] D. H. Fremlin and S. Shelah, On partitions of the real line, Israel J. Math. 32 (1979), 299-304.

[K] K. Kunen, Set Theory, North-Holland, Amsterdam 1980.

$[\mathrm{M}]$ A. W. Miller, Covering $2^{\omega}$ with $\omega_{1}$ disjoint closed sets, in: The Kleene Symposium, J. Barwise, J. Keisler, and K. Kunen (eds.), North-Holland, 1980, 415-421. 
[N] P. J. Nyikos, A supercompact topology for trees, preprint.

[S] W. Sierpiński, Un théorème sur les continus, Tôhoku Math. J. 13 (1918), 300303.

[SV] P. Štěpánek and P. Vopěnka, Decomposition of metric spaces into nowhere dense sets, Comment. Math. Univ. Carolin. 8 (1967), 387-404.

[T] S. Todorčević, Trees and linearly ordered sets, in: Handbook of Set-Theoretic Topology, K. Kunen and J. E. Vaughan (eds.), North-Holland, 1984, 235-293.

[W] S. Watson, Problem Session at the Spring Topology Conference, Univ. of Calif. at Sacramento, April, 1991.

DEPARTMENT OF MATHEMATICS

AUBURN UNIVERSITY

AUBURN, ALABAMA 36849

U.S.A. 